\title{
VITAMIN D SUPPLEMENTATION AND FUNCTIONAL KNEE OSTEOARTHRITIS PROGRESSION IN OLDER ADULTS WITH OBESITY: DATA FROM THE OSTEOARTHRITIS INITIATIVE
}

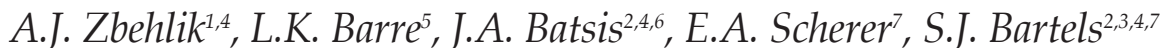

\begin{abstract}
Objective: Older adults with obesity are at increased risk of knee osteoarthritis (KOA) and vitamin D deficiency, but data on the effect of vitamin D supplementation in this population are equivocal. This study evaluated the effect of vitamin $\mathrm{D}$ supplementation on functional progression of KOA in older adults with obesity. Participants with Body Mass Index $\geq 30 \mathrm{~kg} / \mathrm{m} 2$ and aged $\geq 60$ years from the Osteoarthritis Initiative progression cohort were stratified by baseline vitamin D use. The relationship between vitamin D supplementation and progression of KOA at 72 months was characterized. The Western Ontario McMaster University Osteoarthritis Index (WOMAC) pain scale was the primary outcome measure. Secondary measures included: WOMAC disability, Physical Activity Scale for the Elderly, gait speed and Knee injury and Osteoarthritis Outcome Score (KOOS) scales. In older adults with KOA and obesity, baseline supplemental vitamin D use did not predict functional progression of osteoarthritis at 72 months.
\end{abstract}

Key words: Vitamin D, obesity, knee osteoarthritis, older adults.

\section{Introduction}

Knee osteoarthritis (KOA) is a leading cause of disability in the United States and is associated with escalating health care costs. Due to the increasing prevalence of obesity and the aging of the population, the number of knee replacements continues to climb, with a $161 \%$ increase over the past decade (1). One potential low-cost intervention to improve outcomes in $\mathrm{KOA}$ is vitamin $\mathrm{D}$. Vitamin $\mathrm{D}$ is a fat-soluble hormone with multiple effects on bone, cartilage, and muscle: all tissues implicated in the morbidity of $\operatorname{KOA}(2,3)$. Favorable actions on bone turnover and mineralization; cell proliferation and apoptosis; and muscle strength and function are possible mechanisms for vitamin D to improve outcomes in KOA (3). These effects may be mediated through vitamin $\mathrm{D}$ receptors on target tissues, hormone regulation, and immune modulation (3). Yet little is known about the effect of vitamin D

1. Section of Rheumatology, Dartmouth-Hitchcock Medical Center, 1 Medical Center Drive, Lebanon, NH 03756; 2. Centers for Health and Aging, Dartmouth College, Lebanon, NH 03766; 3. The Dartmouth Institute, Dartmouth College, Hanover, NH 03755; 4. Geisel School of Medicine at Dartmouth, Hanover, NH 03755; 5. Division of Nutritional Sciences, Cornell University, Ithaca, NY 14853; 6. Section of General Internal Medicine, Dartmouth-Hitchcock Medical Center, 1 Medical Center Drive, Lebanon, NH, 03756; 7. Department of Community and Family Medicine, Geisel School of Medicine at Dartmouth, Hanover, NH 03755

Corresponding Author: Alicia J. Zbehlik, Division of Rheumatology, DartmouthHitchcock Medical Center, One Medical Center Drive, Lebanon, NH 03756, Phone: 603-650-8622, Fax: 603-650-4961, Email: alicia.j.zbehlik@hitchcock.org supplementation on functional outcomes in older adults with obesity who are at highest risk for KOA and have lower serum vitamin D levels compared to normal weight individuals (4).

Research on the role of vitamin D in improving outcomes in KOA is inconclusive. Several observational studies link low vitamin D intake and serum levels with radiographic progression of $\mathrm{KOA}$, knee pain, and lower functional status (5-8). A 2013 systematic review found that low serum vitamin $\mathrm{D}$ is associated with radiographic progression of disease and vitamin D supplementation may decrease pain scores $(9,10)$. In contrast, a large observational study found no association between vitamin D levels, radiographic progression, or cartilage loss by MRI and a two-year randomized controlled trial found no association between vitamin $\mathrm{D}$ serum levels or supplementation and radiographic OA progression $(11,12)$. Yet none of these studies focused specifically on older adults with obesity, a population that is at high risk for incident and progressive KOA, and may be vitamin $\mathrm{D}$ deficient, in part, due to less sun exposure and highervolumes of distribution (3). Consequently, they may be more dependant on supplements to meet their vitamin $\mathrm{D}$ requirements, and supplementation may therefore be more important in this growing population (3). To address this gap in the literature, this study considered whether older adults with obesity and KOA who report taking vitamin D supplements, compared to those not 
taking vitamin $\mathrm{D}$, have better long-term functional outcomes.

\section{Methods}

Data used in this analysis were from the publically available Osteoarthritis Initiative (OAI) collected at baseline and 72 months. The OAI is a prospective cohort study of KOA that includes incident, prevalent, and control groups. The prevalent cohort includes individuals aged 45-79 years with radiographically confirmed, symptomatic tibiofemoral OA in at least one knee (for detailed methods see www.oai.ucsf.edu). The study was classified as exempt by the Committee for protection of Human Subjects of Dartmouth College. Participants were recruited between February 2004 and May 2006, the 72 month data set was released in February 2013 and analyzed in April 2013. Subjects with obesity $\left(\right.$ BMI $\left.\geq 30 \mathrm{~kg} / \mathrm{m}^{2}\right)$ aged $\geq 60$ years were selected and stratified by baseline self-reported vitamin D use. Vitamin D use included taking a vitamin D supplement alone or with calcium at least once per month to as frequently as daily. Functional disease progression measures included the Western Ontario McMaster University OA Index (WOMAC) pain (primary outcome) and disability scales; Physical Activity Scale for the Elderly (PASE); and gait speed $(\mathrm{m} / \mathrm{s})$; and The Knee injury and Osteoarthritis Outcome Score (KOOS) function, sports, and recreational activities; pain; and quality of life scales. Baseline demographic and clinical characteristics were compared across vitamin D supplementation groups by one-way ANOVA and Pearson's chi-squared test. Vitamin D intake and change in $\mathrm{OA}$ functional progression measures at 72 months was evaluated with multiple separate linear regression analyses adjusting for age, gender, race, and depression (measured by the Center for Epidemiological Studies Depression Scale) as adults with depression and KOA experience more severe pain and activity limitations (13). Due to small numbers, a dichotomous variable compared any vitamin D supplementation to no supplementation. A subset analysis was conducted on women. To evaluate whether the effect of vitamin D in individuals with obesity differed from the effect in normal weight (BMI $\geq 19$ and $<25 \mathrm{~kg} / \mathrm{m}^{2}$ ) and overweight (BMI $\geq 25$ and $<30$ $\mathrm{kg} / \mathrm{m}^{2}$ ), the interaction of normal weight, overweight and obesity and baseline vitamin D supplementation was evaluated using multiple linear regression analysis.

\section{Results}

Older adults with KOA and obesity $(n=352)$ were identified (Table 1). Women ( $p$ 0.001) and whites $(\mathrm{p}<0.001)$ were more likely to take vitamin $\mathrm{D}$ supplements. KOOS left knee pain (p 0.009); KOOS

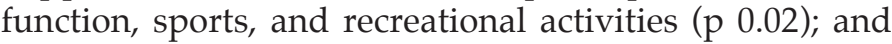

KOOS quality of life scores ( $\mathrm{p}$ 0.01) were significantly different at baseline, with higher scores in the $\geq 5$ year vitamin D supplementation group. WOMAC pain, WOMAC function, KOOS right knee pain, PASE, and gait speed did not differ between the vitamin $\mathrm{D}$ groups at baseline. Table 2 presents the results of multiple linear regression analyses for each of the dependent variables including pain, disability, physical activity, and gait speed six-year follow-up in those taking vitamin D supplements as compared to those not taking vitamin D supplements at baseline. For each of the primary outcome measures there were no differences in disease progression between those taking vitamin $\mathrm{D}$ and those not taking vitamin $\mathrm{D}$. The subset among women, and the interaction model between normal, overweight, and obese individuals and vitamin D supplementation at baseline showed no statistically significant differences in change in dependant variables over 72 months (data not presented).

\section{Discussion}

This study did not show a difference in functional progression of KOA between older adults with obesity who took vitamin D supplements at baseline and those who did not. There are several possible explanations for the lack of observed effect. The volume of distribution of vitamin $\mathrm{D}$ in adults with obesity may lead to less bioavailable vitamin $\mathrm{D}$ to exert an effect in the pathogenesis of $\operatorname{KOA}(2,3)$. It is possible that older adults with obesity may need higher doses of vitamin D to have an effect on functional progression, or the study included subjects with osteoarthritis too advanced to observe a benefit. Finally, it is possible that vitamin D supplementation does not result in a clinically significant benefit in reducing the functional progression of $\mathrm{KOA}$, especially in older adults with obesity.

The findings should be interpreted with caution due to several study limitations. First, vitamin D supplementation was based upon self-report and subject to recall bias. Second, lack of data on total dose and the grouping of monthly vitamin D use with daily use may make the estimates more conservative. Third, only baseline data for vitamin D supplementation was available and prospective use is unknown. Fourth, serum vitamin D levels are not publically available in this data set, so we are unable to tell if participants who supplemented had higher serum vitamin D levels than those who did not. Finally, participants who reported taking vitamin $\mathrm{D}$ differed by race and gender from those who did not supplement, and this may be a source of confounding in this observational study. In this study, African Americans reported lower rates of vitamin D supplementation, and a prior study examining osteoarthritis pain and vitamin D status noted that African Americans may have higher levels of experimentally measured pain associated with vitamin 
Table 1

Characteristics of older adults with obesity in the Osteoarthritis Initiative prevalent cohort

\begin{tabular}{|c|c|c|c|c|}
\hline & \multicolumn{3}{|c|}{ VITAMIN D SUPPLEMENTATION } & \multirow[b]{3}{*}{$\mathbf{P}$} \\
\hline & \multirow{2}{*}{$\begin{array}{c}\text { UNEXPOSED } \\
\text { None }\end{array}$} & \multicolumn{2}{|c|}{ EXPOSED } & \\
\hline & & $<5$ years & $\geq 5$ years & \\
\hline Sample size $(\mathrm{n}, \%)$ & $259(73.6)$ & 49 (13.9) & $44(12.5)$ & NA \\
\hline Age (years) (mean, SD) & $67.2(5.1)$ & $67.4(5.2)$ & $68.5(4.8)$ & 0.31 \\
\hline BMI (kg/m2) (mean, SD) & $34.1(3.1)$ & $33.2(3.2)$ & $33.5(3.7)$ & 0.21 \\
\hline Sex (n,\% female) & $151(58.3)$ & $37(75.5)$ & $34(77.3)$ & 0.001 \\
\hline Race $(\mathrm{n}, \%)$ & & & & $<0.001$ \\
\hline Black & $105(40.5)$ & $14(28.6)$ & $5(11.4)$ & \\
\hline Asian, other non- white & $7(2.7)$ & $3(6.1)$ & $1(2.3)$ & \\
\hline White & $147(56.8)$ & $32(65.3)$ & $38(86.4)$ & \\
\hline CES-D (mean, SD) & $7.5(6.8)$ & $7.0(5.9)$ & $8.9(8.0)$ & 0.36 \\
\hline CCS (mean, SD) & $0.74(1.1)$ & $0.50(0.7)$ & $0.68(1.4)$ & 0.38 \\
\hline \multicolumn{5}{|l|}{ WOMAC (mean, SD) } \\
\hline Pain (left) & $4.5(4.4)$ & $5.2(4.5)$ & $4.2(3.8)$ & 0.50 \\
\hline Pain (right) & $4.9(4.1)$ & $5.4(4.6)$ & $4.7(4.0)$ & 0.65 \\
\hline Function (left) & $16.1(13.7)$ & $19.9(15.3)$ & $14.0(13.3)$ & 0.10 \\
\hline Function (right) & $16.7(13.1)$ & $17.8(13.4)$ & $17.8(13.4)$ & 0.77 \\
\hline PASE (mean, SD) & $123(65)$ & $141(71)$ & $130(73)$ & 0.23 \\
\hline Gait speed (m/sec) (mean, SD) & $1.2(0.3)$ & $1.1(0.2)$ & $1.2(0.2)$ & 0.26 \\
\hline \multicolumn{5}{|l|}{ KOOS (mean, SD) } \\
\hline Knee pain (left) & $76.1(21.1)$ & $70.4(20.5)$ & $79.1(17.5)$ & 0.009 \\
\hline Knee pain (right) & $73.8(19.4)$ & $70.5(19.4)$ & $74.8(19.0)$ & 0.25 \\
\hline $\begin{array}{l}\text { Function, Sports, Recreational } \\
\text { Activities }\end{array}$ & $54.7(25.8)$ & $44.0(26.1)$ & $58.4(25.4)$ & 0.02 \\
\hline Quality of Life & $52.9(19.1)$ & $48.6(15.6)$ & $56.0(18.3)$ & 0.01 \\
\hline
\end{tabular}

BMI=Body Mass Index, CCS=Charlson Co-morbidity Score (range 0-5; higher scores associated with higher 1 year mortality), CES-D=Center for Epidemiologic Studies Depression Scale (range 0-60; higher scores associated with greater depressive symptoms), KOOS=Knee injury and Osteoarthritis Outcome Score (range per scale 0-100; $0=$ extreme knee problems; $100=$ no knee problems), PASE= Physical Activity Scale for the Elderly (range 0-400; higher associated with greater physical activity), SD = Standard Deviation, WOMAC= Western Ontario McMaster University Osteoarthritis Index (ranges: function 0-68, pain 0-20; higher scores worse)

$\mathrm{D}$ deficient states (14). There were also differences in most baseline KOOS scores, with participants taking supplements $\geq 5$ years having higher (better) scores. While there was no change over time, higher scores at baseline in the $\geq 5$ year group may indicate that they are qualitatively different from the other groups.

Despite these limitations, strengths of this study included a focus on the high-risk subgroup of older adults with obesity in a well-defined cohort with prevalent KOA using functional progression measures. We used multiple measures of function to ensure that this negative result was robust. The population was older and had a higher average BMI than participants in the 2013 vitamin D randomized trial (mean age 61.8, mean BMI 30.5 in the vitamin D group) and the 1996 Framingham study where the mean BMI was $26.1 \mathrm{~kg} /$ $\mathrm{m} 2$ and fewer of knees were evaluated $(\mathrm{n}=75)(5,12)$. Finally, the study reflects naturally occurring vitamin D supplementation in a sample of older adults with obesity and KOA supporting generalizability the population as a whole.

In summary, vitamin D supplementation did not alter functional outcomes of KOA in a community sample of older adults with obesity. Future controlled trials may need to consider multi-component approaches to prevention of osteoarthritis in high-risk adults with obesity (15).

\footnotetext{
Funding: This work is supported by the Dartmouth Institute for Health Policy and Clinical Practice and the Department of Medicine, Division of Rheumatology at Dartmouth-Hitchcock Medical Center. The authors are solely responsible for the content.
}

Acknowledgements: «The OAI is a public-private partnership comprised of 
Table 2

Multiple linear regression analysis: Change in dependent variable from baseline to 72 months in those taking vitamin D supplements compared to those not taking vitamin D supplements at baseline

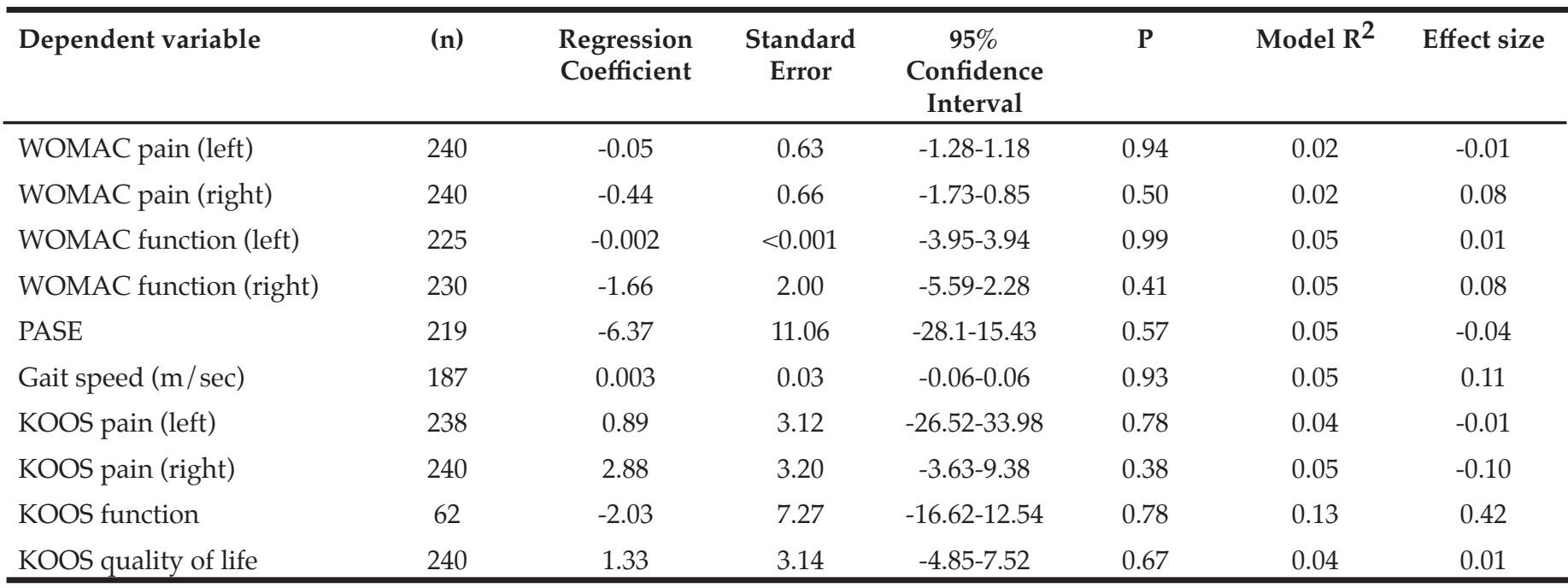

BMI=Body Mass Index, CES-D=Center for Epidemiologic Studies Depression Scale, KOOS=Knee injury and Osteoarthritis Outcome Score, PASE= Physical Activity Scale for the Elderly, WOMAC = Western Ontario McMaster University Osteoarthritis Index

five contracts (N01-AR-2-2258; N01-AR-2-2259; N01-AR-2-2260; N01-AR-2-2261; N01-AR-2-2262) funded by the National Institutes of Health, a branch of the Department of Health and Human Services, and conducted by the OAI Study Investigators. Private funding partners include Merck Research Laboratories; Novartis Pharmaceuticals Corporation, GlaxoSmithKline; and Pfizer, Inc. Private sector funding for the OAI is managed by the Foundation for the National Institutes of Health. This manuscript was prepared using an OAI public use data set and does not necessarily reflect the opinions or views of the OAI investigators, the $\mathrm{NIH}$, or the private funding partners.»

Conflict of Interest Statement: Alicia Zbehlik: Dr. Zbehlik has nothing to disclose relevant to the above work. Laura Barre: Dr. Barre has nothing to disclose relevant to the above work. John Batsis: Dr. Batsis has nothing to disclose relevant to the above work. Emily Scherer: Dr. Scherer has nothing to disclose relevant to the above work. Stephen Bartels: Dr. Bartels reports a CDC Health Promotion Research Center Grant and a HRSA Geriatric Education Center Grant.

Ethical Standards: This study was considered exempt by the Committee for Protection of Human Subjects of Dartmouth College.

\section{References}

1. Cram P, Lu X, Kates SL, Singh JA, Li Y, Wolf BR. Total knee arthroplasty volume, utilization, and outcomes among Medicare beneficiaries, 1991-2010. Jama 2012;308:1227-36.

2. Vanlint S. Vitamin D and obesity. Nutrients 2013;5:949-56.

3. Holick MF. Vitamin D deficiency. The New England journal of medicine 2007;357:266-81

4. Zhang Y, Niu J, Felson DT, Choi HK, Nevitt M, Neogi T. Methodologic challenges in studying risk factors for progression of knee osteoarthritis. Arthritis care \& research 2010;62:1527-32.

5. McAlindon TE, Felson DT, Zhang Y, et al. Relation of dietary intake and serum levels of vitamin D to progression of osteoarthritis of the knee among participants in the Framingham Study. Ann Intern Med 1996;125:353-9.
6. Bergink AP, Uitterlinden AG, Van Leeuwen JP, et al. Vitamin D status, bone mineral density, and the development of radiographic osteoarthritis of the knee: The Rotterdam Study. Journal of clinical rheumatology : practical reports on rheumatic \& musculoskeletal diseases 2009;15:230-7.

7. Muraki S, Dennison E, Jameson K, et al. Association of vitamin D status with knee pain and radiographic knee osteoarthritis. Osteoarthritis and cartilage / OARS, Osteoarthritis Research Society 2011;19:1301-6.

8. Jansen JA, Haddad FS. High prevalence of vitamin D deficiency in elderly patients with advanced osteoarthritis scheduled for total knee replacement associated with poorer preoperative functional state. Annals of the Royal College of Surgeons of England 2013;95:569-72.

9. Cao Y, Winzenberg T, Nguo K, Lin J, Jones G, Ding C. Association between serum levels of 25-hydroxyvitamin D and osteoarthritis: a systematic review. Rheumatology (Oxford, England) 2013;52:1323-34.

10. Sanghi D, Mishra A, Sharma AC, et al. Does vitamin D improve osteoarthritis of the knee: a randomized controlled pilot trial. Clinical orthopaedics and related research 2013;471:3556-62.

11. Felson DT, Niu J, Clancy M, et al. Low levels of vitamin D and worsening of knee osteoarthritis: results of two longitudinal studies. Arthritis and rheumatism 2007;56:129-36.

12. McAlindon T, LaValley M, Schneider E, et al. Effect of vitamin D supplementation on progression of knee pain and cartilage volume loss in patients with symptomatic osteoarthritis: a randomized controlled trial. Jama 2013;309:155-62.

13. Knoop J, van der Leeden M, Thorstensson CA, et al. Identification of phenotypes with different clinical outcomes in knee osteoarthritis: data from the Osteoarthritis Initiative. Arthritis Care Res (Hoboken) 2011;63:1535-42.

14. Glover TL, Goodin BR, Horgas AL, et al. Vitamin D, race, and experimental pain sensitivity in older adults with knee osteoarthritis. Arthritis and rheumatism 2012;64:3926-35.

15. Cao Y, Jones G, Cicuttini F, et al. Vitamin D supplementation in the management of knee osteoarthritis: study protocol for a randomized controlled trial. Trials 2012;13:131. 